form and the patient's name label is visible between the slits. These forms cost 0.26P each and, because the slits are cut during the printing process, no extra cost is involved.

The system was designed specifically for $9.8 \mathrm{~cm}$ long, $1.4 \mathrm{~cm}$ diameter blood tubes which form a large part of the work-load, but in practice it has been found that swabs and other tubes can be used with the forms. Specimens which may be dangerously infected are not transported in the slitted forms. We are currently looking into the possibility of obtaining small polythene bags, approximately $13 \mathrm{~cm}$ long $\times$ $4 \mathrm{~cm}$ wide, into which high-risk specimens may be placed, thus allowing the tube to be wrapped before it is inserted into the form. These small bags should be significantly cheaper than the larger ones currently in use.

Slitted forms have been in use in this hospital since October 1974 and have been well received. It is very unusual for a specimen to become separated from the request form, and the slits do not interfere with the subsequent photo-copying and filing of the reports. The system has the advantage that the doctor is given the opportunity to check that the specimen is labelled when it is inserted in the form. Furthermore, it eliminates the frustration caused by specimens arriving without request forms and vice versa.

\section{A method for distinguishing sheep erythrocytes adherent to human tissue sections}

\author{
R. T. ALLISON, M. SLADE, and D. M. WALKeR Depart- \\ ment of Oral Medicine and Oral Pathology, Welsh \\ National School of Medicine, Dental School, Heath, \\ Cardiff
}

Lymphocyte subpopulations and histiocytes may be identified in frozen sections of human and mouse tissues by means of known differences in cell membrane receptors (Edelson et al, 1973). Briefly, sheep erythrocytes complexed with anti-sheep red cell IgM and mouse complement, or alternatively coated with anti-sheep red cell IgG alone, are layered on frozen sections. Sheep erythrocytes remaining adherent following washing of the treated sections act as particulate markers of complement receptor bearing lymphocytes and IgG receptor bearing histiocytes respectively in the underlying tissue sections.

Phase-contrast and dark-ground microscopy help in the detection of bound erythrocytes, but visualization, particularly photomicrography, has been difficult.

Amido Black B was introduced as a stain for haemoglobin (Puchtler and Sweat, 1962) and experience with this technique has led to its adoption for the demonstration of sensitized erythrocytes adher-

Received for publication 17 July 1975. ing to tissue sections in this laboratory. Puchtler and Sweat claim specificity for the technique. Hodson (1967) believes that the rationale of the technique depends upon the affinity of Amido Black B for the globin moiety of the haemoglobin molecule and reflects structural chemistry rather than a histochemical reaction. The intense blue/black colour produced in erythrocytes lends itself admirably to photomicrography and contrasts well with many histological stains. Small numbers of adherent erythrocytes are easily detected and their tissue localization becomes simple.

The technique needs to be slightly modified for application to the adherent sheep erythrocytes and has been found to perform satisfactorily as follows: 1. Following exposure to the sensitized erythrocytes the tissue sections are washed in phosphate buffered saline and then fixed in $3 \%$ glutaraldehyde for 30 minutes.

2. Wash in phosphate buffered saline.

3. Treat with $5 \%$ tannic acid-10 minutes.

4. Wash in water and treat with freshly prepared $1 \%$ phosphomolybdic acid-20 minutes.

5. Wash in water and shake nearly dry.

6. Treat with a saturated solution of Amido Black 10B (Syr. Naphthalene black) in 9 vol: 1 vol methanol:acetic acid-30 minutes.

7. Rinse in methanol:acetic acid solution and then water.

8. Counterstain in $1 \%$ saffranin -5 seconds.

9. Wash rapidly, dehydrate, clear, and mount.

Sites of adherent erythrocytes are readily visualized and may be distinguished from erythrocytes 


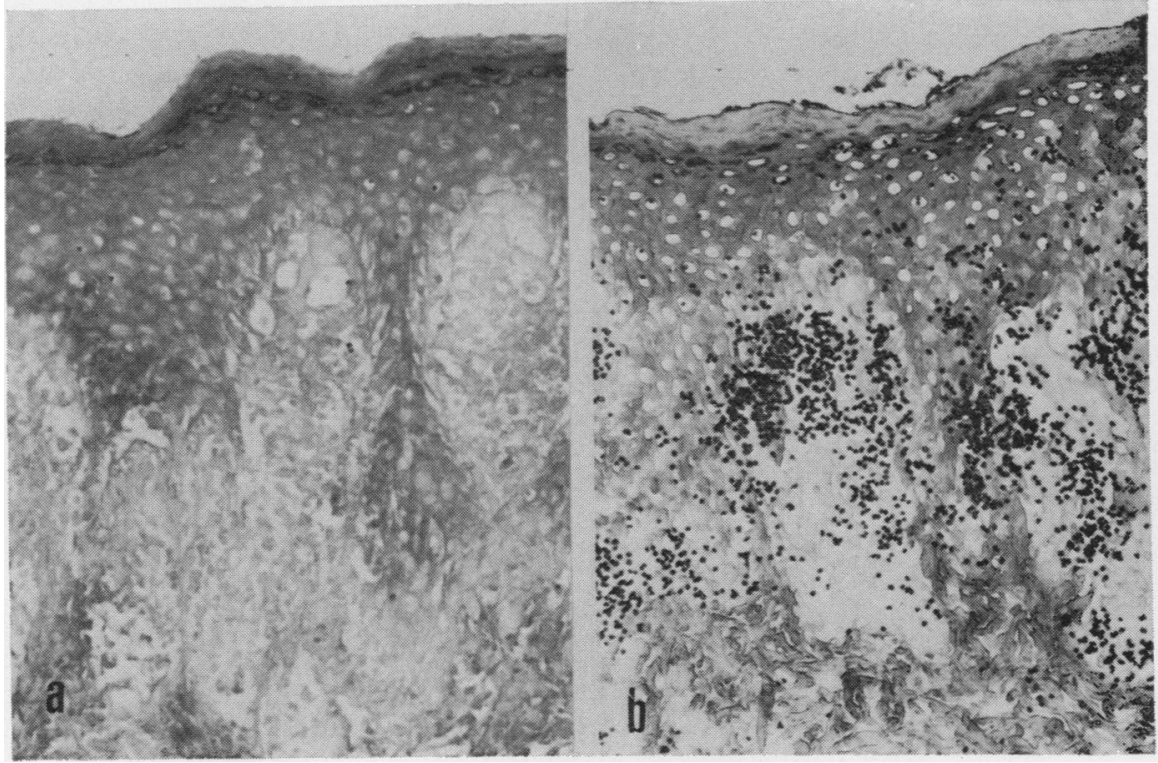

Figure (a) Control section-stained but without sheep erythrocytes; (b) Amido Black staining following exposure of tissue section to 'sensitized' sheep erythrocytes. $\times 30$.

present in the underlying tissue which stain a very pale colour and occur in a different plane of focus.

\section{References}

Edelson, R. L., Smith, R. W., Frank, M. M., and Green, I. (1973). Identification of subpopulations of mononuclear cells in cutaneous infiltrates. $J$. invest. Derm., 61, 82-89.

Hodson, J. J. (1967). The distribution, structure, origin and nature of the dental cuticle of Gottlieb. Periodontics, 5, 237-250.

Puchtler, H. and Sweat, F. (1962). Amido black as a stain for hemoglobin. Arch. Path., 73, 245-249.

\section{Letter to the Editor}

\section{Coulter Counter Model 'S' System}

The Coulter Counter Model ' $\mathrm{S}$ ' system is of undoubted importance in the routine haematology laboratory. Its design is still evolving as improvements to it are continually being made which enhance its reliability and performance. I wish to point out a potential flaw in the design and suggest how it might be corrected.

The self-contained pneumatic unit supplies the operating vacuum and pressures to the diluter unit, which in turn is essen- tially a micropneumatic system designed to give fine control. The micro components (Wade Couplings Ltd) ought to be driven by dry particle-free air. However, in the Coulter S system they are frequently driven by moist air, which may of course contain dissolved, and perhaps corrosive, solutes. (In our instrument crystalline material accumulates in the pressure storage tanks, and at times the pressure transmission tubes have been found to contain water.) Further, the efficiency of the components, and in particular of the shuttle valves that control the mixing and transfer rates, becomes impaired. A typical consequence of the shuttle valves being faulty is shown in the figure. What is not shown is that the fault may take hours to pinpoint $\sigma$ and correct, even by an experienced $N$ engineer.

I suggest that the problem might be minimized or even corrected completelyi by incorporating a drier unit and carbon filter between the compressor and the $\mathbb{D}$ diluter units. Alternatively, a dry air : supply of appropriate pressures from a 0 central source could be employed should this facility exist. Coulter Electronics Ltd suggested that the fault could be corrected by spraying the shuttle valves with a release/lubricating oil. I was not surprised 